\title{
A Path-Following Approach to Stable Bipedal Walking and Zero Moment Point Regulation
}

\author{
D. Djoudi, C. Chevallereau, and J.W. Grizzle
}

\begin{abstract}
Consider a biped evolving in the sagittal plane. The unexpected rotation of the supporting foot can be avoided by controlling the zero moment point or ZMP. The objective of this study is to propose and analyze a control strategy for simultaneously regulating the position of the ZMP and the joints of the robot. If the tracking requirements were posed in the time domain, the problem would be underactuated in the sense that the number of inputs would be less than the number of outputs. To get around this issue, the proposed controller is based on a path-following control strategy previously developed for dealing with the underactuation present in planar robots with unactuated ankles. In particular, the control law is defined in such a way that only the kinematic evolution of the robot's state is regulated, but not its temporal evolution. The asymptotic temporal evolution of the robot is completely defined through a one degree of freedom subsystem of the closed-loop model. Simple analytical conditions, which guarantee the existence of a periodic motion and the convergence towards this motion, are deduced.
\end{abstract}

\section{INTRODUCTION}

The majority of robot control policies are built around the notion of controlling the ZMP point [9], [10]. The center of pressure or $\mathrm{CoP}$ is a standard notion in mechanics that was renamed the zero moment point or ZMP by Vukobratovic and co-workers [12], [11]. As long as the ZMP point remains inside the convex hull of the foot support region, $C o P=$ $Z M P$ and the supporting foot does not rotate. In particular, most of the control strategies are decomposed into a lowlevel controller and a high-level controller, where the lowlevel controller ensures the tracking of the reference motion for each joint, and the high-level controller modifies the reference motion in order to ensure that the ZMP point remains within the convex hull of the foot support region.

The existence and stability of a periodic orbit depend on much more than just the position of the ZMP point: It is quite possible to have gaits where the ZMP point is within the convex hull of the foot support region and where the robot remains upright, but yet the gait is not periodic, or it is periodic, but is not asymptotically stable. In many experimental studies, how to modify the reference motion is not explained [9], and it seems that this point has not been studied theoretically. Obviously, the modification of the reference motion has an important effect on the stability of the gait (in the sense of the convergence toward a periodic motion) and its robustness

D.Djoudi and C. Chevallereau are with the IRCCyN, Ecole Centrale de Nantes, UMR CNRS 6597, BP 92101, 1 rue de la Noë, 44321 Nantes, cedex 03, France, Dalila.Djoudi, Christine.Chevallereau@irccyn.ec-nantes.fr, J.W. Grizzle is with the Control Systems Laboratory, EECS Department, University of Michigan, Ann Arbor, Michigan 48109-2122, USA, grizzle@umich.edu (in the sense of the reaction of the robot in the presence of perturbations).

Our control strategy is based on a path-following control strategy previously developed for dealing with the underactuation present in planar robots without actuated ankles [8], [3], [14], [4]. Our controller is related to the work in [5], which extended the work of Westervelt et al. [14] on underactuated bipedal walking to the case of a fully-actuated robot, where the stance ankle torque was used to regulate either the position of the stance ankle or the rate of convergence to a periodic walking gait. In the present study, the position of the ZMP will instead be prescribed, which is important for robustly avoiding unexpected rotations of the foot in the presence of perturbations.

The control law is defined in such a way that only the geometric evolution of the robot's joints and of the ZMP position is controlled, but not their temporal evolution. This strategy can be seen as an on-line modification of the joint reference motion with respect to time in order to ensure that the position of the ZMP will be satisfactory. The modification of the reference motion corresponds to adjusting the acceleration of the robot along a given path ${ }^{1}$ in the joint space. Assuming a perfect robot model, and without external perturbations, the closed-loop temporal evolution of the robot is completely defined and can be analyzed through the study of a one degree of freedom subsystem. The Poincaré return map can be used to study the existence and stability of periodic motions under the proposed control law. Analytical conditions are obtained and subsequently illustrated through simulations.

Section II presents the dynamic model of the biped. A planar biped is considered. Section III is devoted to the formulation of the control strategy and to the existence of a periodic motion. In Section IV, a complete analytical study is proposed. Some simulation results are presented in Section V. Section VI concludes the paper.

\section{THE BIPED MODEL}

\section{A. The biped}

The biped under study walks in the sagittal plane identified with a vertical $x-z$-plane. The robot is comprised of a torso and two identical legs, and each leg is composed of two links with mass and a foot. The ankles, the knees and the hips are one-degree-of-freedom rotational frictionless joints. The walking gait consists of single support phases where the stance foot is flat on the ground separated by impacts, that

\footnotetext{
${ }^{1}$ The time evolution along the path is not specified a priori. For related work in nonlinear control, see [1] and references therein.
} 
is, instantaneous double support phases where leg exchange takes place. The vector $q=\left[q_{1}, q_{2}, q_{3}, q_{4}, q_{5}, q_{6}\right]^{T}$ of configuration variables (see Fig. 1) describes the shape of the biped during single support. The stance ankle torque, which is used to obtain a desired evolution of the ZMP, is denoted $\Gamma_{a}=\Gamma_{1}$. The torques are grouped into a torque vector $\Gamma=\left[\Gamma_{1}, \Gamma_{2}, \Gamma_{3}, \Gamma_{4}, \Gamma_{5}, \Gamma_{6}\right]^{T}$.

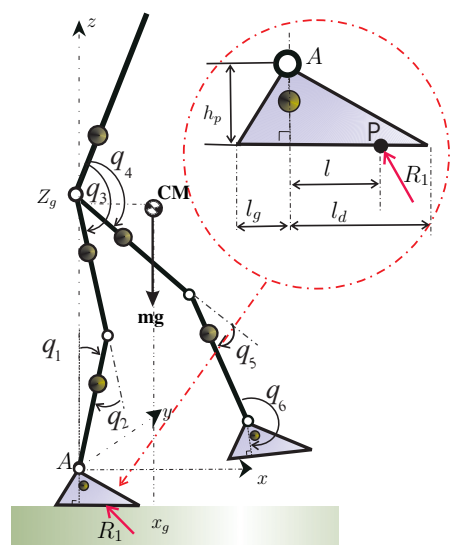

Fig. 1. The studied biped: generalized coordinates

In the simulation, we use the biped parameters given in [3] for all links except the feet. The mass of each foot is $1 \mathrm{~kg}$ and the center of mass is on the normal of the sole passing through the ankle, $3 \mathrm{~cm}$ below the ankle. The dimensions of the feet are $h_{p}=0.08 m, l_{g}=0.06 m$ and $l_{d}=0.2 m$.

\section{B. Dynamic model}

The walking gait is composed of successive phases of single support and instantaneous double support. A passive impact exists at the end of the single support phase. The legs swap their roles from one step to the next, and thus, because the robot is symmetric, the study of a single step is sufficient to deduce the complete behavior of the robot over a sequence of steps on alternating legs. Only the dynamic model for support on leg-1 and the algebraic impact model are derived.

1) The single support phase model: The dynamic model can be written as follows:

$$
M(q) \ddot{q}+h(q, \dot{q})=\Gamma,
$$

where $M(q)$ is a $(6 \times 6)$ matrix and vector $h(q, \dot{q})$ contains the centrifugal, Coriolis and gravity forces.

2) Global equilibrium in translation : The reaction force during the single support phase: During single support, the position of the center of mass of the biped can be expressed as a function of the angular coordinates vector $q$ noted $x_{g}(q)$, $z_{g}(q)$. When leg- 1 is on the ground, a ground reaction force $R_{1}$ exists. The global equilibrium in translation of the robot makes it possible to calculate this force. Thus we have :

$$
m\left[\begin{array}{c}
\ddot{x}_{g} \\
\ddot{z}_{g}
\end{array}\right]+m g\left[\begin{array}{l}
0 \\
1
\end{array}\right]=R_{1}
$$

Equation (2) can also be written:

$$
\begin{aligned}
m \frac{\partial x_{g}(q)}{\partial q} \ddot{q}+m \dot{q}^{T} \frac{\partial^{2} x_{g}(q)}{\partial q^{2}} \dot{q} & =R_{x 1} \\
m \frac{\partial z_{g}(q)}{\partial q} \ddot{q}+m \dot{q}^{T} \frac{\partial^{2} z_{g}(q)}{\partial q^{2}} \dot{q}+m g & =R_{z 1},
\end{aligned}
$$

where $\frac{\partial^{2} x_{g}(q)}{\partial q^{2}}$ and $\frac{\partial^{2} z_{g}(q)}{\partial q^{2}}$ are $(6 \times 6)$ matrices.

3) Global equilibrium in rotation : the ZMP position: The robot is submitted to the reaction force exerted by the ground in the ZMP point, and the gravity force. The equilibrium of the foot around the ankle can be written (see figure 1):

$$
\varsigma_{A}=m g x_{g}-l R_{z 1}-h_{p} R_{x 1} .
$$

where $\varsigma_{A}$ is the resultant moment of the dynamic wrench of the robot about the stance ankle $A$. Since the stance ankle $A$ is fixed during the single support phase, we have $\varsigma_{A}=\dot{\sigma}_{A}$ where $\sigma_{A}$ is the angular momentum of the biped about $A$. Using equations (2), equation (4) becomes:

$$
\dot{\sigma}_{A}=m g x_{g}-l R_{z 1}-h_{p} R_{x 1} .
$$

By definition the angular momentum is linear with respect to the joint velocities and can be written:

$$
\sigma_{A}=N(q) \dot{q}
$$

The location of the ZMP point is then defined directly by the robot dynamics through the previous equation. Indeed, using equations (3) and (6), we have:

$$
\left(N_{0}(q)+l N_{l}(q)\right) \ddot{q}+h_{0}(q, \dot{q})+l h_{l}(q, \dot{q})=0,
$$

where

$$
\begin{aligned}
N_{0} & =N(q)+m h_{p} \frac{\partial x_{g}(q)}{\partial q} \\
N_{l} & =m \frac{\partial z g(q)}{\partial q} \\
h_{0} & =\dot{q}^{T} \frac{\partial N(q)}{\partial q} \dot{q}-m g x_{g}(q)+m h_{p} \dot{q}^{T} \frac{\partial^{2} x_{g}(q)}{\partial q^{2}} \dot{q} \\
h_{l} & =m \dot{q}^{T} \frac{\partial^{2} z_{g}(q)}{\partial q^{2}} \dot{q}+m g
\end{aligned}
$$

Equation (5), can also be rewritten, using (2), in the form

$$
\frac{d\left(\sigma_{A}+l\left(m \dot{z}_{g}\right)+h_{p}\left(m \dot{x}_{g}\right)\right)}{d t}-m i \dot{z}_{g}-m g\left(x_{g}-l\right)=0 \text {. }
$$

By definition, the term $\sigma_{A}+l\left(m \dot{z}_{g}\right)+h_{p}\left(m \dot{x}_{g}\right)$ is the angular momentum about the ZMP point with $x$-coordinate $l$, which is denoted here by $\sigma_{P}$. This equation, corresponding to angular momentum balance, can be also written as

$$
\dot{\sigma}_{P}=m i \dot{z}_{g}+m g\left(x_{g}-l\right) \text {. }
$$

\section{The impact model}

When the swing leg (i.e., leg-2) touches the ground with a flat foot at the end of the single support phase, an impact takes place. The resulting ground reaction is described by a Dirac delta-function with intensity $I_{R_{2}}$. The velocity of foot2 becomes zero just after the impact. We study a gait with instantaneous double support so that during the impact, the stance leg-1 lifts off the ground. The robot's configuration $q$ is assumed to be constant during the instant of double support, while there are jumps in the velocities. The velocity vectors just before and just after impact, are denoted $\dot{q}^{-}$and $\dot{q}^{+}$, respectively, where ${ }^{+}$means after the impact and ${ }^{-}$before the impact. The impact model can be written as [4]:

$$
\dot{q}^{+}=E\left(\triangle(q) \dot{q}^{-}\right),
$$

where $\triangle(q)$ is a $6 \times 6$ matrix, and $E$ is the permutation matrix describing leg exchange. 
The single support phase corresponds to $0<s<1$. Due

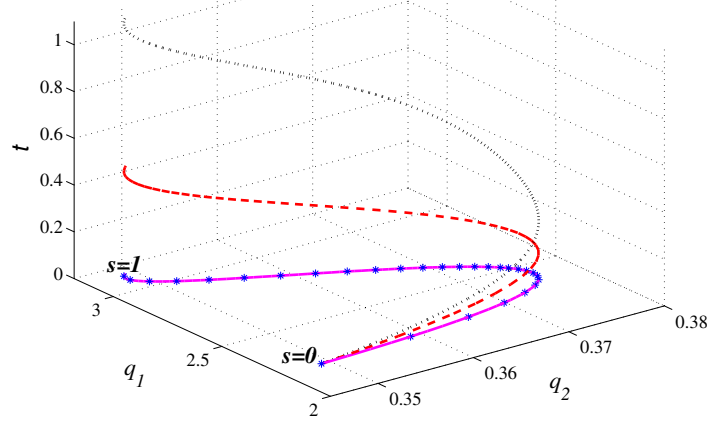

Fig. 2. The dotted lines are two motions $\left(q_{1}(t), q_{2}(t)\right)$ corresponding to the same path represented by the solid line. A path is a line in the joint space, this line can be graduated as a function of a new variable denoted $s$, and then can be expressed by $\left(q_{1}(s), q_{2}(s)\right)$. This function $s$ is defined such that the initial configuration correspond to $s=0$, the final configuration corresponds to $s=1$. Any monotonic function $s(t)$ defines a motion corresponding to the path $q(s)$. For example $s=t / T$ defines a motion of duration $T$.

\section{The CONTROL Strategy}

The desired walking gait is assumed to be composed only of single support phases where the stance foot is flat on the ground and stationary (i.e., it does not slip). While a flat-footed gait is not a necessary condition for walking, and as shown in [5], rotation about the toe can be easily included in the analysis of the control strategy, we focus our attention on fully-actuated phases. Direct control of the position of the ZMP point will prevent unwanted foot rotation, and thus a desired evolution, $l^{d}$, is prescribed [7]. As shown in the previous section, the position of the ZMP is directly connected to the acceleration of the robot's motion. It is therefore impossible to prescribe independently a desired evolution of the joints, $q^{d}(t)$, and of the position of the ZMP, $l^{d}(t)$. With respect to such a task, the biped can be seen as an underactuated system. Thus as in [4], the objective of the control law presented in this section is not to track a (time-based) reference motion for $q$ and $l$, but only the associated path in joint space. A reference motion differs from a path by the fact that a motion is a temporal evolution along a path. A joint path is the projection of a joint motion in the joint space. The difference between a motion and a path is illustrated in Fig. 2 for a two-joints robot.

Only tracking of the desired path is sought, and a timescaling control law as in [6] is used. A reference joint and ZMP paths $q^{d}(s)$ and $l^{d}(s)$ are assumed to be known as a function of a scalar path parameter $s$, which plays the role of a normalized virtual time. The desired gait of the robot corresponds to specification of $s$ as an increasing function of time, $s(t)$.

\section{A. Requirements for a Feasible Reference Path}

The reference path $q^{d}(s), l^{d}(s)$ is designed in order to be compatible with a periodic solution of the biped model. The legs swap their roles from one step to the next, so the reference path can be defined for one step only. For the first step, the scalar path parameter $s$ increases strictly monotonically with respect to time from 0 to 1 and impact takes place at $s=1$. The evolution of $s$ along the step $k$ is denoted $s_{k}(t)$. to the leg exchange at impact, the vectors $q^{d}(0)$ and $q^{d}(1)$, describing respectively the initial and final desired positions of the biped, must be such that $q^{d}(1)=E\left(q^{d}(0)\right)$.

The initial and final velocity of the biped are connected by the impact model and leg exchange (10). The reference path is designed so that if the reference path is exactly tracked before the impact (but the robot state is not necessary on the periodic motion), then the reference path will be exactly tracked after the impact. Just before the $k+1$-st impact, on the reference path, the vector of joint velocities is $\dot{q}^{-}=\frac{d q^{d}(1)}{d s} \dot{s}_{k}(1)$. The reference path is designed such that, after the impact, the reference path is also perfectly tracked: $\dot{q}^{+}=\frac{d q^{d}(0)}{d s} \dot{s}_{k+1}(0)$. Since the impact model (10) connects the velociticies before and after impact we must have

$$
\frac{d q^{d}(0)}{d s} \dot{s}_{k+1}(0)=E \triangle\left(q^{d}(1)\right) \frac{d q^{d}(1)}{d s} \dot{s}_{k}(1) .
$$

When $q^{d}(1)$ and $\frac{d q^{d}(1)}{d s}$ are known, there is an infinite number of possible choices for $\frac{d q^{d}(0)}{d s}$. The set of solutions can be parametrized by a scalar $\alpha$ as

$$
\frac{d q^{d}(0)}{d s}=E \triangle\left(q^{d}(1)\right) \frac{d q^{d}(1)}{d s} \alpha,
$$

yielding $\dot{s}_{k+1}(0)=\frac{\dot{s}_{k}(1)}{\alpha}$.

\section{B. Definition of the control law}

The control law is selected to ensure that the joint coordinates follow the joint reference path, $q^{d}(s)$, and that the position of the ZMP is $l^{d}(s)$. The torque acts on the second derivative of $q$ and directly on $l$. It follows from the definition of the joint reference path that the desired velocity and acceleration of the joint variables are:

$$
\begin{aligned}
& \dot{q}^{d}(t)=\frac{d q^{d}(s(t))}{d s} \dot{s} \\
& \ddot{q}^{d}(t)=\frac{d q^{d}(s(t))}{d s} \ddot{s}+\frac{d^{2} q^{d}(s(t))}{d s^{2}} \dot{s}^{2}
\end{aligned}
$$

The function $s(t)$ needs to be a strictly increasing function of $t$, but since the control objective is only to track a reference path, the evolution $s(t)$ is otherwise free and the second derivative $\ddot{s}$ can be treated as a "supplementary control input". This allows the control law to be designed for a system with equal number of inputs and outputs: The control inputs are the six torques $\Gamma_{j}, j=1, \ldots, 6$, plus $\ddot{s}$, and the chosen outputs are the six components of $q(t)-q^{d}(s(t))$ and $l(t)-l^{d}(s(t))$.

The control law is based on computed torque, which is quite commonly used in robotics, with a small modification to ensure finite-time convergence to the desired paths. The finite-time feedback function proposed in [2], [8] is used. The joint tracking errors are defined with respect to trajectories satisfying (13)

$$
\begin{aligned}
& e_{q}(t)=q^{d}(s(t))-q(t) \\
& \dot{e}_{q}(t)=\frac{d q^{d}(s(t))}{d s} \dot{s}-\dot{q}(t) .
\end{aligned}
$$

The desired behavior of the configuration variables in closed loop is

$$
\ddot{q}=\ddot{q}^{d}+\psi(q, \dot{q}, s, \dot{s},)
$$


where $\psi(q, \dot{q}, s, \dot{s}$,$) from [2], [8] is the term that imposes$ $\left(q(t)-q^{d}(s(t))\right) \rightarrow 0$ in finite time; in fact, it can be chosen to be less than the time duration of a step. Taking into account the expression for the reference motion, equation (15) can be rewritten as:

$$
\ddot{q}=\frac{d q^{d}(s)}{d s} \ddot{s}+v(s, \dot{s}, q, \dot{q}),
$$

with $v(s, \dot{s}, q, \dot{q})=\frac{d^{2} q^{d}(s)}{d s^{2}} \dot{s}^{2}+\psi$. For the position of the ZMP, the desired closed-loop behavior is:

$$
l(t)=l^{d}(s(t))
$$

Combining expression (16) with the dynamic model (1) of the robot and the relation (7) for the ZMP determines the feedback controller. Thus the control law must be such that

$$
\begin{aligned}
M(q)\left(\frac{d q^{d}(s)}{d s} \ddot{s}+v\right)+h(q, \dot{q}) & =\Gamma \\
\left(N_{0}(q)+l^{d}(s) N_{l}(q)\right)\left(\frac{d q^{d}(s)}{d s} \ddot{s}+v\right) & \\
+h_{0}(q, \dot{q})+l^{d}(s) h_{l}(q, \dot{q}) & =0 .
\end{aligned}
$$

It follows that, in order to obtain the desired closed-loop behavior, it is necessary and sufficient to choose

$$
\begin{aligned}
\ddot{s} & =\frac{-\left(N_{0}(q)+l^{d}(s) N_{l}(q)\right) v-h_{0}(q, \dot{q})-l^{d}(s) h_{l}(q, \dot{q})}{\left(N_{0}(q)+l^{d}(s) N_{l}(q)\right) \frac{d^{d}\left(q^{d}\right)}{d s}} \\
\Gamma & =M(q)\left(\frac{d q^{d}(s)}{d s} \ddot{s}+v\right)+h(q, \dot{q}) .
\end{aligned}
$$

As long as $\left(N_{0}(q)+l^{d}(s) N_{l}(q)\right) \frac{d q^{d}(s)}{d s} \neq 0$, the control law (18) is well defined, and, by (15), ensures that, $q(t)$ converges to $q^{d}(s(t))$ in finite time, and that $l(t)=l^{d}(s(t))$.

\section{Stability StUdy}

Since the occurrence of an impact depends only on the configuration of the robot and not its velocity, and due to the characteristics of the joint reference path (section III-A), each step begins with $s=0$ and finishes with $s=1$. Since the control law is designed to converge before the end of the first step, after that, perfect tracking is obtained and therefore

$$
\begin{aligned}
& q(t)=q^{d}(s(t)) \\
& \dot{q}(t)=\frac{d q^{d}(s)}{d s} \dot{s}(t) \\
& \ddot{q}(t)=\frac{d q^{d}(s)}{d s} \ddot{s}(t)+\frac{d^{2} q^{d}(s)}{d s^{2}} \dot{s}(t)^{2} \\
& l(t)=l^{d}(s(t)) .
\end{aligned}
$$

These equations define the zero dynamics manifold corresponding to the proposed control law. On the zero dynamics manifold, the evolution of $\dot{s}$ during one step can be determined by integration of the dynamic equation corresponding to the global equilibrium in rotation (9).

\section{A. Evolution of the angular momentum for one step}

On the zero dynamics, equation (9) becomes :

$$
\dot{\sigma}_{P}=m \frac{d l^{d}(s)}{d s} \frac{\partial z_{g}\left(q^{d}(s)\right)}{\partial q} \frac{d q^{d}(s)}{d s} \dot{s}^{2}+m g\left(x_{g}\left(q^{d}(s)\right)-l^{d}(s)\right)
$$

and

$$
\sigma_{P}(s, \dot{s})=I(s) \dot{s}
$$

with

$I(s)=\left(N\left(q^{d}(s)\right)+m l^{d}(s) \frac{\partial z_{g}\left(q^{d}(s)\right)}{\partial q}+m h_{p} \frac{\partial x_{g}\left(q^{d}(s)\right)}{\partial q}\right) \frac{d q^{d}(s)}{d s}$.

Equations $(20,21)$ can be combined to express the derivative of the angular momentum with respect to $s$.

Just as in [14, Prop. 1], it can be shown that uniqueness of solutions of $(20,21)$ implies that if the robot completes a step, that is, if there exists a solution beginning with $s(0)=0$, $\dot{s}(0)>0$ and ending with $s\left(t_{f}\right)=1, \dot{s}\left(t_{f}\right)>0$, then for $t \in\left[0, t_{f}\right], \dot{s}(t)>0$. Using this fact, equations $(20,21)$ can be combined to express:

$$
\begin{aligned}
\frac{d \sigma_{P}}{d s}= & m \frac{d l^{d}(s)}{d s} \frac{\partial z_{g}\left(q^{d}(s)\right)}{\partial q} \frac{d q^{d}(s)}{d s} \frac{\sigma_{P}}{I(s)} \\
& +m g\left(x_{g}\left(q^{d}(s)-l^{d}(s)\right) \frac{I(s)}{\sigma_{P}} .\right.
\end{aligned}
$$

Applying the change of variable, $\zeta(s)=\frac{1}{2} \sigma_{P}^{2}$, this equation becomes:

$$
\frac{d \zeta}{d s}=2 \kappa(s) \zeta+m g I(s)\left(x_{g}\left(q^{d}(s)-l^{d}(s)\right) .\right.
$$

with $\kappa(s)=\frac{m}{I(s)^{2}} \frac{d l^{d}(s)}{d s} \frac{\partial z_{g}\left(q^{d}(s)\right)}{\partial q} \frac{d q^{d}(s)}{d s}$. The above equation is a linear s-varying ODE and has the explicit solution:

$$
\zeta(s)=\delta^{2}(s) \zeta(0)-\Phi(s)
$$

where

$$
\begin{aligned}
& \delta(s)=\exp \left(\int_{0}^{s} \kappa(\tau) d \tau\right) \\
& \Phi(s)=-2 m g \int_{0}^{s} \exp \left(2 \int_{\tau}^{s} \kappa\left(\tau_{1}\right) d \tau_{1}\right) I(\tau)\left(x_{g}\left(q^{d}(\tau)\right)-l^{d}(\tau)\right) d \tau .
\end{aligned}
$$

\section{B. Minimal angular momentum to achieve a step}

The functions $\delta(s)$ and $\Phi(s)$ are calculated directly from $q^{d}(s)$ and $l^{d}(s)$. A complete step can be accomplished only if $\dot{s}$ is always positive. With the assumption that the reference trajectory is such that $\left(N_{0}\left(q^{d}(s)\right)+l^{d}(s) N_{l}\left(q^{d}(s)\right)\right) \frac{d q^{d}(s)}{d s}=$ $I(s) \neq 0$. The condition $\dot{s} \neq 0$ is equivalent to $\sigma_{P} \neq 0$ or $\zeta \neq 0$.

Theorem 1 A step can be achieved if and only if the initial value of $\zeta$ for this step is such that

$$
\zeta(0)>Z_{m}=\max _{0 \leq s \leq 1}\left(\frac{\Phi(s)}{\delta^{2}(s)}\right) .
$$

\section{Evolution of angular momentum during the impact phase}

At the impact, due to equation (12), the evolution of $\dot{s}$ is such that $\dot{s}^{+}=\frac{\dot{s}^{-}}{\alpha}$. Thus

$$
\sigma_{P}^{+}=\delta_{I} \sigma_{P}^{-}
$$

with $\delta_{I}=\frac{I(0)}{I(1) \alpha}$, where $I$ is given in equation (22). 


\section{Conditions of existence and uniqueness of periodic motion}

The combination of equations (25) and (27) defines the evolution of $\zeta$ (or, equivalently, $\sigma_{P}$ ) from one step to the next. The evolution of the robot during one step is completely defined by the value of $\zeta$ for one value $s$. Thus, we study the evolution of $\zeta$ just before the impact $\zeta^{-}=\zeta(1)$ from one step to the next, via the Poincaré map

$$
\rho\left(\zeta^{-}\right)=\left(\delta_{I} \delta(1)\right)^{2}\left(\zeta^{-}\right)-\Phi(1) .
$$

A periodic admissible reference motion is defined by a periodic evolution of the angular momentum, which is equivalent to a fixed point of the Poincaré map $\rho: \zeta^{*}=\rho\left(\zeta^{*}\right)$.

¿From equation (28), taking into account that $\zeta(s)>0$, it follows that:

- if $\left(\delta_{I} \delta(1)\right)^{2}=1$ and $\Phi(1)=0$, then any initial value $\zeta$ produces a periodic reference motion.

- the poincaré map has a unique fixed point

$$
\zeta^{*}=\frac{-\Phi(1)}{1-\left(\delta_{I} \delta(1)\right)^{2}},
$$

if, and only if, $\Phi(1)$ and $1-\left(\delta_{I} \delta(1)\right)^{2}$ have different signs.

Applying Theorem 1 and using (28), $\zeta^{*}$ in (29) defines a periodic reference motion if, and only if, the periodic angular momentum is sufficient to produce the step: $\left(\delta_{1}\right)^{2} \zeta^{*}>Z_{m}$.

Theorem 2 A unique periodic reference motion exists if, and only if $\frac{-\Phi(1)}{1-\left(\delta_{I} \delta(1)\right)^{2}}>\frac{Z_{m}}{\left(\delta_{I}\right)^{2}}$. The periodic motion is defined by equation (29).

\section{E. Convergence Towards the Cyclic Reference Motion}

Equation (28) is equivalent to

$$
\rho\left(\zeta^{-}\right)-\zeta^{*}=\left(\delta_{I} \delta(1)\right)^{2}\left(\zeta^{-}-\zeta^{*}\right) .
$$

Consequently, solutions of equations $(20,21)$ converge to the periodic motion if, and only if, $\left(\delta_{I} \delta(1)\right)^{2}<1$.

Theorem 3 Solutions of the zero dynamics given by equations $(20,21)$ converge to the periodic reference motion if and only if $\left(\delta_{I} \delta(1)\right)^{2}<1$.

Combining theorems 1,2 and 3 , the following corollary can be deduced.

Corollary: The reference periodic motion is orbitally exponentially stable if, and only if, the reference joints path is such that: $-\Phi(1)>\max \left(\frac{1-\left(\delta_{I} \delta(1)\right)^{2}}{\delta_{I}^{2}} Z_{m}, 0\right)$ and $\left(\delta_{I} \delta(1)\right)^{2}<1$.

\section{Simulation RESUlts}

The control law is evaluated here for the periodic path depicted in the stick-diagram of Fig. 3. The joint path $q^{d}(s)$ is defined with a degree four polynomial in $s$. The evolution of the ZMP position is chosen to be a linear function of $s$. As $s$ varies from 0 to $1, l^{d}$ will vary from $-0.02 m$ to $0.08 m$.

The reference trajectory $l^{d}(s), q^{d}(s)$ can be the result of an optimisation process since the cyclic motion, if it exists, can be explicitly deduced via (29). A methodology allowing to do this is given in [13].

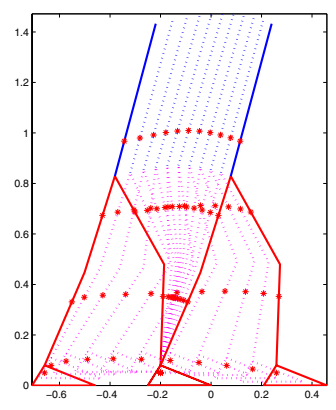

Fig. 3. The stick diagram of the desired trajectory. The configuration of the robot is drawn for $s=0,0.1,0.2 \ldots, 0.9,1$. Thus a sequence of pictures of the robot is given. The desired motions of the robot are such that the configuration of the robot coincides at some instant to each picture, but it is not imposed that these instants are equally distributed within the period of one step.
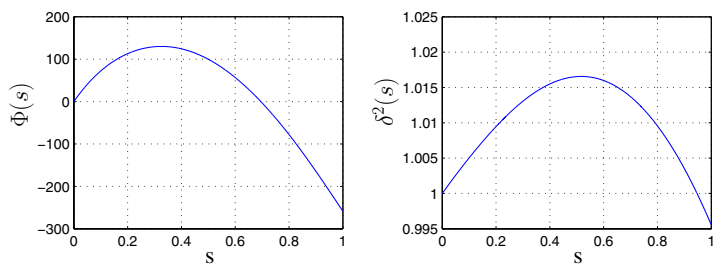

Fig. 4. The evolution of $\zeta$ during the single support phase is characterized by the functions $\Phi(s)$ and $\delta^{2}(s)$. The position of the ZMP point is being controlled to increase linearly, from back to front. Because the vertical component of the velocity of the center of mass is directed upward at the beginning of the step and then downward, $\delta(s)$ increases at the beginning of the step and then decreases. Because the center of mass is behind the ZMP point at the beginning of the step and then in front of the ZMP point, $\Phi(s)$ increases at the beginning of the step and then decreases.

The evolution of $\delta(s)$ and $\Phi(s)$ are given in Fig. 4. Their final values, $\Phi(1)=-205$, and $\delta(1)^{2}=0.9954$ are useful for constructing the Poincaré map. The behavior of $\zeta$ during the impact is defined by $\delta_{I}^{2}$, which is equal to 0.6422 . The minimal value of $\zeta$ for which a step can be achieved is $\frac{Z_{m}}{\delta_{I}}=232$. The periodic motion is given by equation (29). The fixed point occurs at $\zeta^{*}=569$. The slope of the Poincare return map $\rho$ is $\left(\delta(1) \delta_{I}\right)^{2}=0.6393$, and because it is less than 1 , the corresponding periodic walking motion is exponentially stable. The stability arises from the effect of the impact because $\delta(1)^{2}$ is close to 1 .
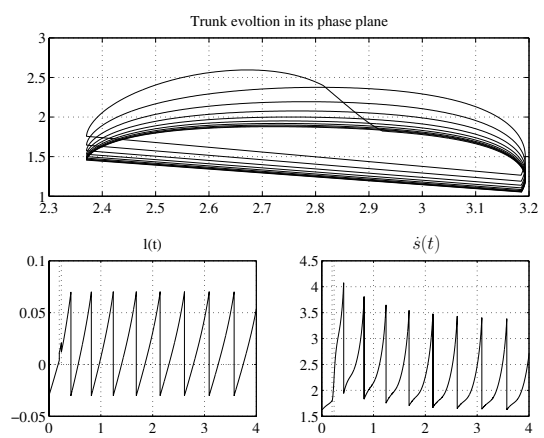

Fig. 5. The convergence towards a periodic motion is observed in simulation with the proposed control law.

A simulation was done for ten steps, assuming no modeling 
error and initializing the state of the robot on the periodic orbit, a horizontal force $(350 \mathrm{~N})$ is applied for $0.2 s<t<0.24 s$ at the center of mass; see Fig. 5. Convergence toward a periodic motion was obtained for each of the five joints of the robot. As an illustration, the evolution of the angle of the torso is depicted in Fig. 5-a. The same convergence is also evident in the evolution of the position of the ZMP point with respect to time in Fig. 5-b; for each step, its evolution is linear from $-0.02 m$ to $0.08 m$ except when the perturbation exists. Fig. 5-c presents the evolution of $\dot{s}$ with respect to time; it clearly converges toward a periodic motion. This control approach is also robust with respect to modeling error [13].

\section{A. Effect of the ZMP evolution}

\begin{tabular}{|c|c|c|c|c|c|c|}
\hline$l_{\min }$ & $l_{\max }$ & $\zeta^{*}$ & $\frac{Z_{m}}{\delta_{I}^{2}}$ & $\left(\delta(1) \delta_{I}\right)^{2}$ & $\dot{s}_{c}(1)$ & $T[s]$ \\
\hline-0.05 & 0.05 & 943.02 & 149.69 & 0.613 & 3.86 & 0.401 \\
-0.04 & 0.06 & 823.92 & 175.44 & 0.621 & 3.62 & 0.437 \\
-0.03 & 0.07 & 699.48 & 202.78 & 0.630 & 3.36 & 0.488 \\
-0.02 & 0.08 & 569.18 & 231.61 & 0.639 & 3.04 & 0.569 \\
-0.01 & 0.09 & 432.40 & 261.85 & 0.648 & 2.66 & 0.733 \\
0 & 0.10 & 288.45 & 293.42 & \multicolumn{5}{|c|}{ No Cyclic motion } \\
\hline
\end{tabular}

TABLE I

THE EFFECT OF THE ZMP EVOLUTION

The evolution of the ZMP point throughout the step affects the existence and stability of the periodic motion obtained with the proposed control law. To illustrate this point, we consider various linear evolutions of the position of the ZMP point with different average values, $\left(\frac{l^{d}(1)+l^{d}(0)}{2}\right)$ while holding constant the net change in the position of the ZMP point, $l^{d}(1)-l^{d}(0)=$ $10 \mathrm{~cm}$. Table I presents the main properties of the periodic motion and of the control law with respect to the variation of the average value of the ZMP position during one step. Placing the average position of the ZMP closer to the toe
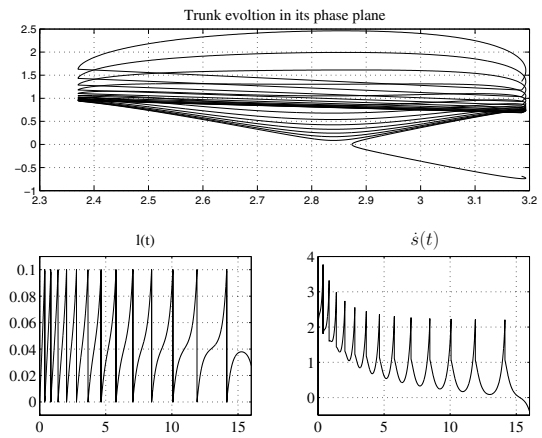

Fig. 6. The motion of the robot is not stable, it does not converge to a periodic motion, but the position of the ZMP remains inside of the sole of the stance feet.

leads to larger values of $\left(\delta(1) \delta_{I}\right)^{2}$ and smaller values of $\zeta^{*}$ and average walking speed. When the center of mass is in front of the ZMP point, the moment arm due to gravity speeds up the motion. When the center of mass is behind the ZMP point, the moment arm due to gravity slows down the motion. When the average position of the ZMP is moved forward, the portion of the step where gravity speeds up the motion decreases, and thus the average walking speed decreases.

In the last row of table I, the value of $\zeta^{*}$ is less than the minimum value necessary to complete a step (i.e., it does not satisfy equation (26)), and consequently a walking motion cannot be produced. If the control law is used for this case, the behavior shown in Fig. 6 is obtained. A perfect tracking of the joint path is observed, the position of the $Z M P$ satisfies at each time the condition of non rotation of the feet (fig 6 left lower part) but the motion of the robot is not stable. The robot does not fall down but it comes to a stop.

\section{CONCLUSION}

For a planar biped, a control strategy was proposed based on tracking a reference path in the joint space instead of a reference function of time. This allows the simultaneous control of the path positions of the joints and the ZMP. The biped adapts its time evolution according to the effect of gravity. A stability study of the robot's time evolution has been presented. Easily testable analytical conditions have been presented for the existence and uniqueness of a periodic motion for the orbital exponential stability of a periodic motion.

\section{ACKNOWLEDGMENTS}

The work of D. Djoudi and C.Chevallereau was supported by ANR grants for the PHEMA project. The work of J.W. Grizzle was supported by a National Science Foundation grant, ECS 0600869.

\section{REFERENCES}

[1] A.P. Aguiar, J.P. Hespanha, and P.V. Kokotovic. Path-following for nonminimum phase systems removes performance limitations. IEEE Transactions on Automatic Control, 50(2):234 - 239, 2005.

[2] S. P. Bhat and D. S. Bernstein. Continuous finite-time stabilization of the translational and rotational double integrators. IEEE Transaction on Automatic Control, 43(5):678-682, 1998.

[3] C. Chevallereau, G. Abba, Y. Aoustin, F. Plestan, E. R. Westervelt, C. Canudas-de Witt, and J. W. Grizzle. Rabbit: A testbed for advanced control theory. IEEEControl System Magazine, 23(5):57-78, 2003.

[4] C. Chevallereau, Formal'sky A. M., and D. Djoudi. Tracking of a joint path for the walking of an under actuated biped. Robotica, 22(1):15-28, 2004.

[5] Jun Ho Choi and J.W. Grizzle. Planar bipedal walking with foot rotation. In $A C C, 2005$.

[6] O. Dahl and L. Nielsen. Torque-limited path following by online trajectory time scaling. IEEE Trans. on Automat. Contr, 6(5):554-561, 1990.

[7] D. Djoudi and C. Chevallereau. Fast motions in Biomechanics and Robotics, chapter Stability analysis of bipedal walking with control or monitoring of the center of pressure, pages 95-120. LNCIS. Springer, Heidelberg, Allemagne, 2006.

[8] J. W. Grizzle, G. Abba, and F. Plestan. Asymptotically stable walking for biped robots: analysis via systems with impulse effects. IEEE Trans. on Automat. Contr., 46:51-64, 2001.

[9] K. Hirai, M. Hirose, Y. Haikawa, and T. Takenaka. The development of honda humanod robot. In Proc. of the IEEE International Conference on Robotics and Automation, pages 1321-1326, Leuven, Belgium, 1998.

[10] F. Pfeiffer, K. Loffler, and M. Gienger. The concept of jogging johnie. In IEEE Int. Conf. on Robotics and Automation, 2002.

[11] M. Vukobratovic and B. Borovac. Zero moment point -thirty five years of its live. Int. Journal of Humanoid Robotics, 1(1):157-173, 2004.

[12] M. Vukobratovic, B. Borovac, D. Surla, and D. Stokic. Biped Locomotion. Springer-Verlag, 349p, 1990.

[13] E.R. Westervelt, J.W. Grizzle, C. Chevallereau, J.H. Choi, and B. Morris. Feedback Control of Dynamic Bipedal Robot Locomotion. Taylor and Francis/CRC Press, 2007.

[14] E.R. Westervelt, J.W. Grizzle, and D.E. Koditschek. Hybrid zero dynamics of planar biped walkers. IEEE Transaction on Automatic Control, 48(1):42-56, 2003. 